\section{Relação da ginecomastia puberal com o índice de massa corporal em amostra de adolescentes atendidos em Unidade de Pacientes Externos de Hospital Universitário}

\author{
The relation between pubertal gynecomastia and body \\ mass index in a sample of adolescents attended at the \\ Outpatient Health Unit of a University Hospital
}

Nádia F. Rivera', Evelyn Eisenstein', Cláudia B. M. A. Cardoso²

\section{RESUMO}

Objetivo: Avaliar a associação entre a ginecomastia puberal e o índice de massa corporal (IMC) por idade. Métodos: Uma amostra de 109 casos de indivíduos com idades de 11 a 19 anos foi selecionada na unidade ambulatorial de adolescentes do Hospital Universitário Pedro Ernesto, no período de 2003 a 2006. Os dados antropométricos coletados foram: peso, altura, diâmetro mamário e estágio de maturação sexual. A análise do IMC por idade foi baseada no NHANES I e nos pontos de corte de Cole para sobrepeso e obesidade. 0 nível de significância foi de $\mathrm{p}<$ 0,005. Resultados: A porcentagem de adolescentes com sobrepeso e obesidade foi de $50,4 \%$. O diâmetro mamário aumentou de 1,5 $\pm 1,0$ a 4,86 $\pm 2,20 \mathrm{~cm}$, mostrando correlação significante com os percentis do IMC por idade (Pearson =0,59). Conclusões: $O$ estudo confirmou a correlação significante do diâmetro mamário com os percentis de IMC, demonstrando a associação entre a ginecomastia puberal e IMC mais altos. Arq Bras Endocrinol Metab. 2009;53(4):435-9.

Descritores

Adolescência; antropometria; ginecomastia; obesidade; índice de massa corporal

Abstract

Objective:To evaluate the association between pubertal gynecomastia and body mass index for age. Methods: A sample of 109 cases was selected from the adolescent outpatient health unit, with ages between 11 and 19 years old at Pedro Ernesto University Hospital. Anthropometric measures of weight, height, mammary horizontal diameter and sexual maturation stage were collected. The analysis of the body mass index for age was based on the NHANES I and cutt-off points of Cole. The significance level was $p<0.005$. Results: The percentage of adolescents with overweight and obesity was $50.4 \%$. The mammary diameters increased from $1.5 \pm 1$ to $4.86 \pm$ $2.20 \mathrm{~cm}$, with significant correlation to body mass index for age (Pearson $=0.59$ ). Conclusions: The study confirmed significant correlation of mammary diameters to BMI percentiles and demonstrated that pubertal gynecomastia is associated with a higher IMC. Arq Bras Endocrinol Metab. 2009;53(4):435-9.

Keywords

Adolescence; anthropometry; gynecomastia; obesity; body mass index
Núcleo de Estudos da Saúde do Adolescente (NESA) Hospital Universitário Pedro Ernesto, Universidade do Estado do Rio de Janeiro (UERJ), Rio de Janeiro, RJ, Brasil ' Faculdade de Ciências Médicas, UERJ, Rio de Janeiro, RJ, Brasil

2 Universidade Federal do Rio de Janeiro (UFRJ), Rio de Janeiro, RJ, Brasil

Correspondência para: Nádia Ferreira Rivera Av. Genaro de Carvalho, 2.880, ap. 302 - Recreio dos Bandeirantes 22795-078 - Rio de Janeiro, RJ, Brasi

nadiameddoc@gmail.com

Recebido em 6/Jun/2007 Aceito em 14/Jan/2009

\section{INTRODUÇÃO}

A ginecomastia puberal é definida como o desenvolvimento do tecido glandular mamário do homem durante o período de maturação sexual (1-2). Esse evento é observado precocemente na puberdade, cerca de seis meses após o início do aumento do volume testicular e do surgimento dos pelos pubianos (3). A prevalência em adolescentes normais é de até 75\% (4). O diagnóstico 
diferencial mais comum é a lipomastia, na qual existe acúmulo de tecido adiposo palpável na região mamária sem haver disco de consistência elástica subareolar, correspondendo à proliferação ductal e estromal, presentes na ginecomastia $(1-3,5)$.

Ambas as mamas são afetadas de forma sequencial em 95\% dos casos (1) e a duração habitual é de seis meses a dois anos $(3,4)$, acompanhando a fase do estirão puberal. A involução é espontânea na maioria dos casos e o volume glandular costuma restringir-se ao perímetro areolar. Cerca de $4 \%$ dos adolescentes apresentam formas graves de ginecomastia puberal com diâmetros horizontais de $4 \mathrm{~cm}$ (macromastia) ou maiores de $5 \mathrm{~cm}$ (macroginecomastia) (3). Nesses casos, os adolescentes apresentam mamas que se assemelham às femininas em estágios 3 e 4 de Tanner (6) e não costuma haver regressão $(1,3)$.

A fisiopatologia da ginecomastia puberal é de natureza multivariada, sujeita a influências de origem genética e ambiental. A explicação mais frequente é que, no início da puberdade, a concentração sérica de estradiol (E2), que estimula o desenvolvimento mamário, triplica em relação aos níveis de E2 da criança. A testosterona $(\mathrm{T})$, que antagoniza esse efeito, só alcança níveis adultos, 30 vezes maiores que os níveis pré-puberais, ao final da puberdade. Esse desequilíbrio transitório entre os níveis de E2 e de T provocaria o surgimento da ginecomastia (1-5).

Outra explicação reconhecida diz respeito ao papel da enzima aromatase, responsável pela biossíntese do E2 que, em situações nas quais se encontra elevada, promove o estímulo para a ginecomastia (1-2,5,7-8).

A transcrição genética da aromatase é controlada por promotores tissulares específicos. Estados como obesidade, resistência insulínica e casos descritos de mutação afetando o gene da aromatase apresentam alta incidência de ginecomastia (7-9). Níveis elevados de insulina suprimem a expressão hepática da globulina carreadora dos hormônios sexuais, provocando aumento nos androgênios biodisponíveis. Sua conversão em estrona (E1) e E2 no tecido adiposo levaria à ginecomastia (10). A possibilidade de maior sensibilidade a níveis circulantes normais de E2 também foi descrita como causa de ginecomastia, explicada pela maior expressão tissular da aromatase nos tecidos desses pacientes (2). Pesquisas recentes sugerem que a elevação fisiológica do hormônio luteinizante $(\mathrm{LH})$ na puberdade agiria em receptores de gonadotropinas no tecido mamário masculino. O efeito do LH sobre a mama masculina diminuiria a expressão de receptores androgênicos, o que impediria a ação inibitória da T, contribuindo para o desenvolvimento da ginecomastia (11).

A administração exógena de hormônio do crescimento $(\mathrm{GH})$ também pode provocar o aparecimento de ginecomastia. Questiona-se, contudo, a sua influência, assim como do fator de crescimento insulina-símile (IGF-1), ambos em ascensão na puberdade, na etiologia da ginecomastia puberal (5)

Diante da relevância dada ao papel da obesidade entre os fatores associados à ginecomastia puberal, o presente estudo objetivou estudar a influência desse estado nutricional no desenvolvimento do aumento mamário nos adolescentes da amostra selecionada.

\section{PACIENTES E MÉTODOS}

Foi realizado um estudo descritivo, transversal, de amostra de 109 casos de ginecomastia puberal, consecutivamente selecionados entre 143 adolescentes com idade entre 11 e 19 anos, atendidos no ambulatório do Serviço de Atenção Secundária, no Núcleo de Estudos da Saúde do Adolescente (NESA), do Hospital Universitário Pedro Ernesto (UERJ), no período de outubro de 2003 a junho de 2006. A amostra foi composta por pacientes encaminhados para tratamento de ginecomastia por unidades de saúde do município do Rio de Janeiro e de outros municípios do Estado, assim como por encaminhamento interno do NESA e de outros serviços do hospital. Qualquer massa palpável subareolar foi considerada para avaliação no estudo. O critério de inclusão foi o diagnóstico clínico de ginecomastia puberal, feito pela mesma pesquisadora em todos os pacientes, por meio da história, do exame físico e da avaliação do estágio de maturação sexual pela inspeção e palpação da genitália segundo critérios de Tanner. Os critérios de exclusão foram o uso de qualquer medicamento, de maconha (não houve casos de uso de outras drogas), suspeita de hipogonadismo, hepatopatia, nefropatia, doenças da tireoide, suspeita de síndrome de Cushing, suspeita de síndrome de Klinefelter e casos de lipomastia. Selecionaram-se os pacientes com o diagnóstico de ginecomastia puberal. Todos os participantes eram estudantes, atendidos gratuitamente no Sistema Único de Saúde, sem distinção de classe social.

A avaliação da ginecomastia foi realizada medindose o diâmetro horizontal das mamas, estando o adolescente em posição supina, com as mãos trançadas atrás da cabeça, de frente para o examinador. A mão do 
examinador palpava a mama, fazendo deslizar os dedos em direção ao mamilo. $\mathrm{O}$ disco de tecido elástico, subareolar, não aderido a planos profundos, era pinçado entre o polegar e o indicador e tracionado suavemente, medindo-se seu diâmetro horizontal (2).

Utilizou-se régua milimetrada e a leitura foi realizada com precisão de $0,5 \mathrm{~cm}$.

O diâmetro mamário foi estratificado em faixas de até $1,9 \mathrm{~cm}$, de 2 a $3,9 \mathrm{~cm}$ e de $4 \mathrm{~cm}$ ou maiores. A lipomastia foi diagnosticada e excluída do estudo pela ausência de tecido mamário à palpação.

Os dados antropométricos foram obtidos e analisados seguindo-se as recomendações do comitê da OMS, usando-se como referência o National Health and Nutrition Examination Survey (NHANES) I (12-13) para a classificação de IMC por idade para o sexo masculino - percentil 85 a menor que 95 para risco de sobrepeso e 95 ou maior para obesidade. Para confirmar sobrepeso e obesidade, os dados foram comparados com a tabela de pontos de corte de IMC por idade para adolescentes do sexo masculino de Cole (12-15). A medida do peso corpóreo foi realizada com os adolescentes descalços, despidos de agasalhos, bonés e adereços, em balança digital eletrônica Filizola com medição em kg. A estatura foi aferida em régua antropométrica vertical, estadiômetro de Harpenden, estando o adolescente em posição ereta, com os calcanhares próximos e postura alinhada. A leitura foi realizada com a precisão de 0,1 $\mathrm{cm}$. O IMC foi calculado como a razão da massa corporal $(\mathrm{kg})$ pelo quadrado da estatura $\left(\mathrm{m}^{2}\right)$. O percentil menor do que 5 foi considerado anormal, magreza ou baixo IMC por idade. A maturação sexual foi avaliada de acordo com os critérios de Tanner durante o exame físico (6). A duração da ginecomastia foi calculada por inquérito recordatório, e a referência à mastalgia no início da ginecomastia colaborou para maior precisão na coleta dessa informação.

Para a análise estatística, foi utilizado o programa de computador SPSS versão 11.0 para Windows. Os dados descritivos foram expressos como média \pm desvio-padrão ou como porcentagem. A correlação entre o percentil de IMC por idade e o diâmetro mamário foi calculada mediante o valor do $\mathrm{r}$ de Pearson.

Todos os pacientes e seus responsáveis assinaram o termo de consentimento livre e esclarecido para participação no estudo, o qual foi previamente aprovado pelo Comitê de Ética em Pesquisa do HUPE, seguindo-se as recomendações da Declaração de Helsinki de 1975, conforme revisão de 2000, para pesquisas com seres humanos.

\section{RESULTADOS}

A média de idade encontrada nos adolescentes que participaram do estudo foi de $15 \pm 1,9$ anos, variando de 11 a 19 anos. Em relação à maturação sexual, conforme os critérios de Tanner, encontrou-se maior número de casos nos estágios de desenvolvimento sexual 3 e 4 (Tabela 1).

\begin{tabular}{|c|c|c|c|}
\hline $\begin{array}{l}\text { Estágios de } \\
\text { Tanner G }\end{array}$ & $\begin{array}{c}\text { Número de } \\
\text { pacientes (\%) }\end{array}$ & $\begin{array}{l}\text { Média de idade } \\
\quad \text { (anos) }\end{array}$ & $\begin{array}{l}\text { Diâmetro } \\
\text { mamário médio } \\
\text { (cm) }\end{array}$ \\
\hline 2 & $14(11)$ & $13,0 \pm 1,0$ & $2,8 \pm 1,5$ \\
\hline 3 & $42(38)$ & $14,3 \pm 1,5$ & $3,8 \pm 2,3$ \\
\hline 4 & $29(28)$ & $15,3 \pm 1,4$ & $3,3 \pm 2,3$ \\
\hline 5 & $24(23)$ & $16,9 \pm 1,8$ & $4,1 \pm 2,0$ \\
\hline Total & 109 (100) & $15,0 \pm 1,9$ & $3,6 \pm 2,2$ \\
\hline
\end{tabular}

A avaliação do IMC relacionado à idade revelou $50 \%$ de prevalência de excesso de peso, sendo encontrados 19 adolescentes $(17,4 \%)$ nas faixas de percentis 85 a 95 , e 36 (33\%) acima do percentil 95, o que corresponde a sobrepeso e obesidade, tanto nas curvas da OMS-NHANES I, como nos pontos de corte de Cole. A frequência do baixo IMC por idade ou faixa do percentil abaixo de 5 foi de cinco casos $(4,6 \%)$ (Tabela 2 ).

\begin{tabular}{|c|c|c|}
\hline Percentis & Número de pacientes & Percentua \\
\hline$<5$ & 5 & 4 \\
\hline 5 & 9 & 8 \\
\hline 15 & 13 & 12 \\
\hline 50 & 27 & 25 \\
\hline$\geq 85 a<95$ & 19 & 17 \\
\hline$\geq 95$ & 36 & 33 \\
\hline
\end{tabular}

Fonte: Referência baseada no primeiro National Health and Nutrition Examination Survey (NHANES I) nos Estados Unidos da América (WHO, 1995).

A distribuição dos pacientes em relação ao diâmetro mamário revelou 25 adolescentes $(23 \%)$ na faixa até $1,9 \mathrm{~cm}, 36(33 \%)$ com diâmetros de 2 a $3,9 \mathrm{~cm}$, e 29 (27\%) com mamas de 4 a $5,9 \mathrm{~cm}$. Observaram-se, ainda, 19 adolescentes (17\%) com diâmetros de $6 \mathrm{~cm}$ ou maiores, semelhantes à mama feminina nos estágios 3 e 4 de Tanner. O diâmetro mamário médio foi de 3,7 $\pm 2,2 \mathrm{~cm}$. 
A correlação entre o IMC por idade e a média do diâmetro mamário apresentou significância estatística. Os valores médios dos diâmetros mamários mostraram alta correlação com os percentis de IMC por idade (Pearson $=0,59)$ (Figura 1 ).

Acantose nigricans foi observada no exame físico de 19 pacientes $(17,4 \%)$, entre os quais 18 apresentavam sobrepeso e obesidade. Em dez desses casos os diâmetros mamários eram iguais ou superiores a $4 \mathrm{~cm}$.

Quanto à duração da ginecomastia, a amostra teve 19 adolescentes $(17,4 \%)$ com relato de até seis meses, $14(12,8 \%)$ de seis meses a um ano, $17(15,5 \%)$ de um a dois anos e 13 casos (12\%) de dois a quatro anos. Houve 33 pacientes (30\%) que já apresentavam o quadro há mais de quatro anos.

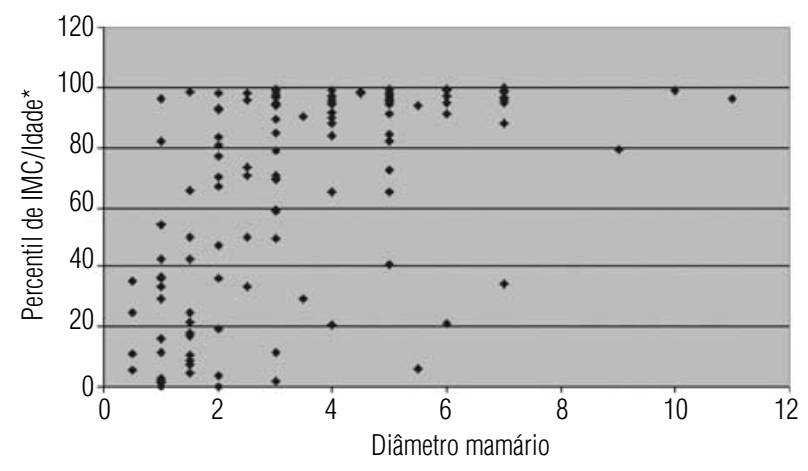

*NHANES I

Cálculo utilizado: $r$ de Pearson =0,590

Figura 1. Correlação entre o diâmetro mamário médio e as curvas do percentil do IMC por idade.

\section{DISCUSSÃO}

O estudo procurou correlacionar a ginecomastia puberal ao IMC por idade por meio das características clínicas e dos dados antropométricos coletados. As considerações quanto aos achados, bem como o que reporta a literatura a respeito, serão feitas a seguir.

\section{Idade}

Os 109 adolescentes selecionados apresentaram média de idade de $15 \pm 1,9$ anos, faixa etária mais avançada, portanto, do que a esperada, segundo Neinstein (1), que avalia que 19,6\% dos adolescentes de 10,5 anos apresentam ginecomastia, com máxima prevalência de 64\% aos 14 anos, diminuindo progressivamente. Observou-se que os pacientes se consultaram com idade acima da esperada, apesar de a amostra concentrar casos mais graves, como característica de hospitais universitários e de referência. Tal resultado também pode ser devido à resolução espontânea, comum aos casos de menores diâmetros, ou por estarem relacionados a estágios de maturação sexual mais avançados, como será mostrado a seguir (Tabela 1 ).

\section{Estágio de maturação sexual de acordo com critérios de Tanner}

O estudo revelou o menor número de pacientes (11\%) no estágio 2 de Tanner, considerado o de maior incidência da ginecomastia puberal, correspondendo a $50 \%$ dos casos, conforme descrito na literatura (1). O maior número de pacientes (38\%) encontrava-se no estágio 3 de Tanner, e o percentual esperado para esse estágio é de $20 \%$ (1). Outra consideração diz respeito à média de idade no estágio 3 (14,3 $\pm 1,5$ anos), maior que o descrito pela OMS para G3 no Brasil, que é de 12,4 anos (12) (Tabela 1).

Conforme já explanado anteriormente, o número relevante e a gravidade dos casos de ginecomastia puberal nos estágios finais de maturação sexual chamam a atenção. A demora para se definirem condutas será abordada no tópico seguinte.

\section{Avaliação do IMC relacionado à idade em relação ao diâmetro mamário encontrado}

A ginecomastia pode ser considerada um risco relacionado à obesidade e ocorrer durante o desenvolvimento da resistência insulínica em crianças e adolescentes, nos quais o ganho excessivo de peso e o hiperinsulinismo precedem o quadro de diabetes resistente à insulina (tipo 2) por vários anos (8). A amostra estudada permitiu observar o número relevante de adolescentes obesos e em sobrepeso (a maioria dos casos, $55 \%$ ) e a gravidade da ginecomastia nesse grupo, em que diâmetros mamários crescentes apresentam correlação significativa, do ponto de vista estatístico ( $\mathrm{r}$ de Pearson $=0,59)$, ao percentil do IMC por idade (Figura 1).

$\mathrm{O}$ excesso de peso encontrado serve também como alerta para a tendência ao incremento de massa corporal para a estatura durante a adolescência e para o aumento da obesidade e suas repercussões sobre a saúde dos adolescentes brasileiros nas últimas décadas (16). O estudo demonstra que a ginecomastia puberal de maiores diâmetros deve ser considerada mais uma morbidade relacionada à obesidade no sexo masculino (Tabela 2). $\mathrm{O}$ agravamento progressivo da ginecomastia puberal, 
provocado pelo sobrepeso e pela obesidade, por meio dos diversos mecanismos propostos aliado à dificuldade de se definir a conduta em adolescentes ainda em processo de maturação sexual, pode provocar impactos negativos sobre a formação da imagem corporal.

Apenas cinco pacientes $(4,6 \%)$ encontravam-se abaixo do percentil 5 para IMC por idade, quadro considerado de desnutrição, responsável pelo atraso do desenvolvimento puberal, mas que pode ser recuperado pela realimentação. Nesses adolescentes desnutridos, a ginecomastia puberal costuma surgir na fase de realimentação e recuperação, sendo autolimitada (17).

\section{Acantose nigricans}

Embora não seja patognomônica, observou-se que esse estigma de resistência insulínica se associou à obesidade e a maiores diâmetros da ginecomastia na amostra estudada, na qual 10 dos 19 pacientes apresentavam diâmetros de $4 \mathrm{~cm}$ ou maiores. $\mathrm{O}$ grau de escurecimento das dobras cutâneas e a extensão do acometimento não foram documentados.

\section{Duração da ginecomastia}

Os adolescentes do estudo apresentaram a ginecomastia por um período que variou de seis meses (19 adolescentes) a mais de quatro anos (33 casos). Os casos mais prolongados comprometeram gravemente toda a adolescência dos pacientes. O surgimento das mamas, um símbolo de feminilidade, os atingiu de maneira negativa, com importantes repercussões sobre a imagem corporal e o desenvolvimento psicossocial. A duração prolongada da ginecomastia puberal torna evidente o lapso entre a deteç̧ão do problema e a definição da conduta diante do caso. O profissional de saúde deve estar atento ao curso arrastado da ginecomastia puberal de maiores diâmetros, especialmente quando associada à obesidade, que poderá funcionar como fator agravante e de cronificação do problema.

Agradecimentos: os autores agradecem a toda a equipe multiprofissional do NESA, aos pacientes e suas famílias, cuja paciência e sensibilidade foram essenciais para superar as dificuldades e limitações encontradas.

Declaração: os autores declaram não haver conflitos de interesse científico neste artigo.

\section{REFERÊNCIAS}

1. Neinstein LS, Joffe A. Gynecomastia. In: Neisntein LS, editor. Adolescent health care, a practical guide. Philadelphia: Lippincott Williams \& Wilkins; 2002. p. 264-70.

2. Braunstein GD. Gynecomastia. N Engl J Med. 1993;328(7): 490-5.

3. Coates V. Evolução histórica da medicina de adolescente. In: Coates V. Medicina do adolescente. $2^{a}$ ed. São Paulo: Sarvier; 2003.

4. Styne D. Puberty. In: Greenspan FS, Gardner DG, editores. Basic and clinical endocrinology. 7th ed. New York: McGraw-Hill Companies; 2004. p. 608-36.

5. Pearlman GDO, Carlson HE. Gynecomastia: an update. Endocrinologist. 2006;16(2):109-15.

6. Tanner JM. Growth at adolescence. 2. ed. Oxford: Blackwell Scientific Publications; 1962.

7. Dunger DB, Ahmed ML, Ong KK. Effects of obesity on growth and puberty. Best Prac Res Clin Endocrinol Metab. 2005;19(3):375-90.

8. Ten S, MacLaren N. Insulin resistance syndrome in children. J Clin Endocrinol Metab. 2004;89(6):2526-39.

9. Shozu M, Sebastian S, Takayama K, Hsu WT, Schultz RA, Neely $\mathrm{K}$, et al. Estrogen excess associated with novel gain-of-function mutations affecting the aromatase gene. $\mathrm{N}$ Engl $\mathrm{J}$ Med. 2003;348(19):1855-65.

10. Chia DJ, Boston BA. Childhood obesity and the metabolic syndrome. Adv Pediatrics. 2006;53:23-53.

11. Carlson HE, Kane $\mathrm{P}$, Lei ZM, Li X, Rao CV. Presence of luteinizing hormone/human chorionic gonadotropin receptors in male breast tissues. J Clin Endocrinol Metab. 2004;89(8):4119-23.

12. World Health Organization. Physical status: the use and interpretation of anthropometry. World Health Organ Tech Rep Ser. 1995;854:1-452.

13. Must A, Dallal GE, DietzWH. Reference data for obesity: 85 th and 95th percentiles of body mass index $\left(w t / h t^{2}\right)$ and triceps skinfold thickness. Am J Clin Nutr. 1991;53:839-46.

14. Cole TJ, Bellizzi M, Flegal KM, Dietz WH. Establishing a standard definition for child overweight and obesity worldwide: international survey. BMJ. 2000;320(6):1240-43.

15. Giugliano R, Melo ALP. Diagnóstico de sobrepeso e obesidade em escolares: utilização do índice de massa corporal segundo padrão internacional. J Ped. 2004;80(2):129-34.

16. Anjos LA, Castro IRR, Engstron EM, Azevedo AMF. Crescimento e estado nutricional em amostra probabilística de escolares no $\mathrm{Mu}$ nicípio do Rio de Janeiro, 1999. Cad Saude Publica 2003;19/Supl 1):171-9.

17. Eisenstein E. Desnutrição crônica primária e atraso puberal. [Tese doutorado]. Escola Paulista de Medicina, Universidade Federal de São Paulo; 1999. 